UCRL-ID-123450

\title{
Study of Electron-Capture Delayed Fission in Am-232
}

\author{
Steven A. Kreek \\ Howard L. Hall \\ Daniel Strellis \\ RECEIVED \\ APR 051996 \\ OSTI \\ Kenneth E. Gregorich \\ Darleane C. Hoffman
}

March 18, 1996

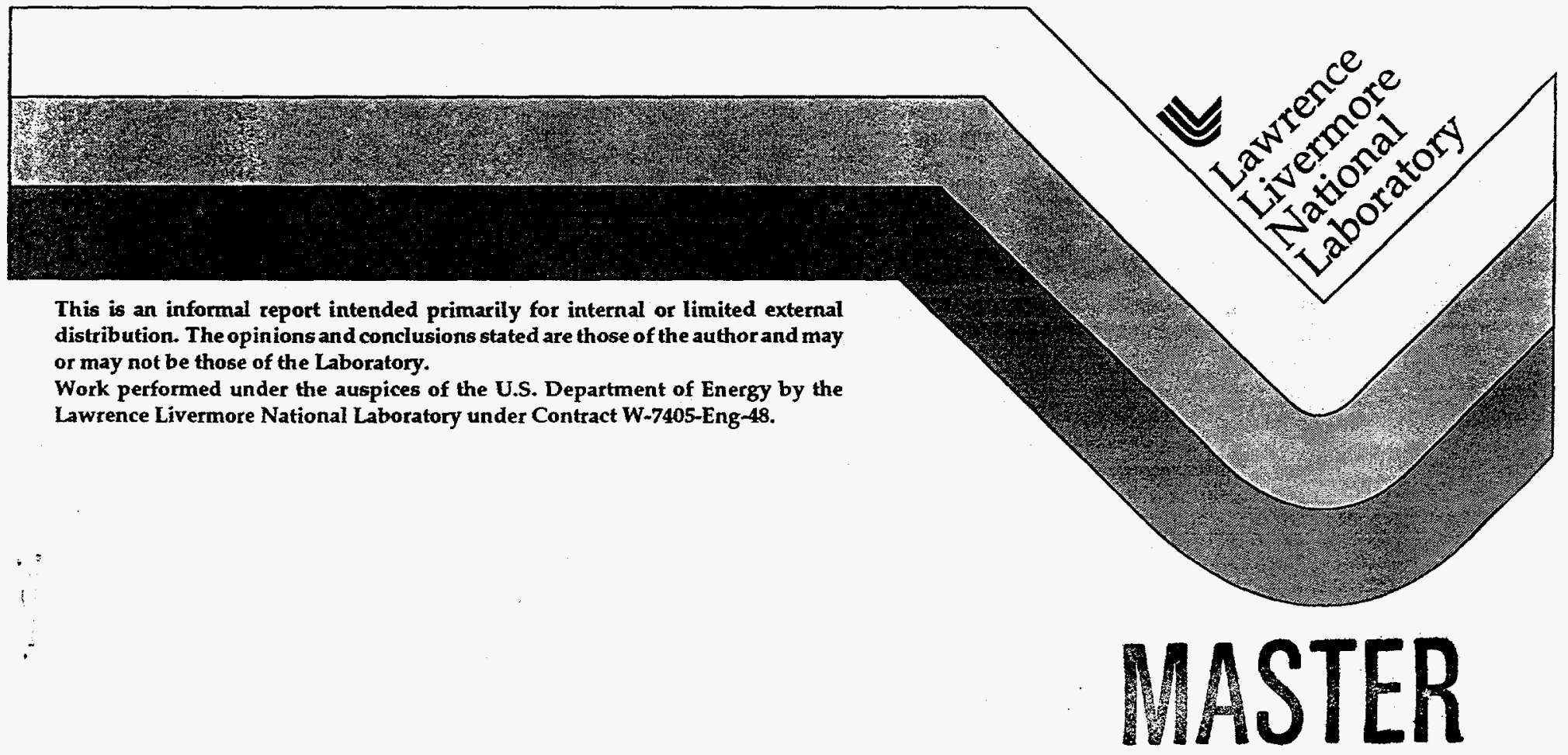


This document was prepared as an account of work sponsored by an agency of the United States Government. Neither the United States Government nor the University of California nor any of their employees, makes any warranty, express or implied, or assumes any legal liability or responsibility for the accuracy, completeness, or usefulness of any information, apparatus, product, or process disclosed, or represents that its use would not infringe privately owned rights. Reference herein to any specific commercial products, process, or service by trade name, trademark, manufacturer, or otherwise, does not necessarily constitute or imply its endorsement, recommendation, or favoring by the United States Govemment or the University of California. The views and opinions of authors expressed herein do not necessarily state or reflect those of the United States Government or the University of California, and shall not be used for advertising or product endorsement purposes.

This report has been reproduced directly from the best available copy.

Available to DOE and DOE contractors from the Office of Scientific and Technical Information P.O. Box 62, Oak Ridge, TN 37831

Prices available from (615) 576-8401

Available to the public from the National Technical Information Service

U.S. Department of Commerce 5285 Port Royal Rd., Springfield, VA 22161 


\section{DISCLAMMER}

Portions of this document may be illegible in electronic image products. Images are produced from the best available original document. 
Study of Electron-Capture Delayed Fission in Am-232

\author{
Steven A. Kreek ${ }^{\dagger}$, Howard L. Hall*, Daniel Strellis ${ }^{\ddagger}$, Kenneth E. Gregorich ${ }^{\not \prime}$, \\ and Darleane C. Hoffman ${ }^{\dagger}$ \\ ${ }^{\dagger}$ Isotope Sciences Division/Glenn T. Seaborg Institute for Transactinium Science, \\ Lawrence Livermore National Laboratory \\ *Analytical Sciences Division, Lawrence Livermore National Laboratory \\ ${ }^{\ddagger}$ Department of Nuclear Engineering, University of California, Berkeley \\ "Nuclear Science Division, Lawrence Berkeley National Laboratory
}

\begin{abstract}
:
An automated $x$-ray-fission coincidence system was designed and constructed by LLNL and Lawrence Berkeley National Laboratory (LBNL) for use inside the Gammasphere high efficiency gamma-ray detector array at LBNL. The x-ray-fission coincidence apparatus detection station consists of two surface barrier detectors (for detection of fission fragments) and two high-purity $\mathrm{Ge}$ (HPGe) planar x-ray detectors (for measurement of $\mathbf{x}$-rays and low-energy gamma rays). The detection station is placed inside Gammasphere at the 88-Inch Cyclotron at LBNL and used in conjunction with Gammasphere to measure the $\mathrm{x}$-rays, low-energy gamma-rays and fission fragments resulting from the ECDF process. A series of collaborative experiment between LLNL, LBNL, and LANL utilizing various components of the $x$-ray-fission coincidence apparatus to measure $\mathrm{x}$-rays and gamma-rays in the decay of a stationary ${ }^{252} \mathrm{Cf}$ source were performed to test the various components of the $x$-ray-fission coincidence apparatus. The test experiments have been completed and the data is currently being analyzed by LBNL. Preliminary test results indicate that the system performed better than expected (e.g., the $\mathrm{x}$ ray detectors performed better than expected with no evidence of microphonic noise that would reduce the photon energy resolution).
\end{abstract}


Introduction:

ECDF is an exotic nuclear decay process in which a nucleus decays via electroncapture to excited states in its daughter nucleus which then fission. These excited states can be above the fission barrier(s) of the daughter (yielding prompt fission), inside the second well of the potential energy surface (a fission shape isomer) or within the first well of the potential energy surface (an electromagnetic isomer). This is illustrated in Figure 1.

For ECDF to become an important mode of deexcitation, the electron-capture Qvalue (QEC) (the energy released during the electron-capture decay) must be comparable to the height of the fission barrier [Hall, 1990A]. If the precursor nuclide chosen for study has a QEC smaller than the fission barrier, the EC would be expected to predominantly populate levels in the first potential well (see Figure 1). Population of states in the second well would require a significant change in deformation to occur simultaneously with the EC decay, and thus is far less probable.

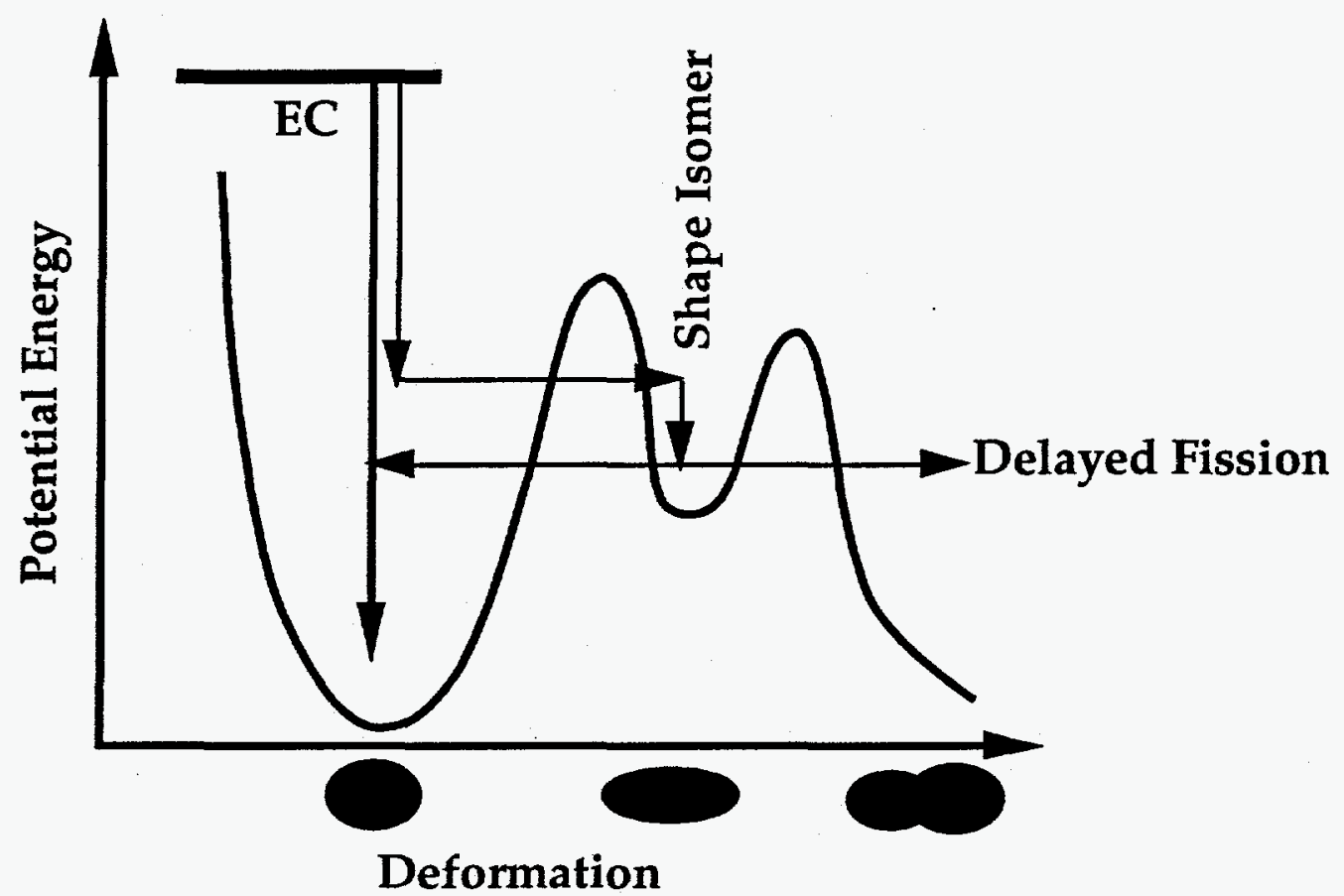

Figure 1. Two-dimensional illustration of the delayed fission process.

The ECDF mode of decay is of interest because of its similarity to beta-delayed fission (BDF), which is believed to have an important role in determining the yields of heavy isotopes produced in multiple neutron capture processes such as nucleosynthesis in the r-process of supernovae and nuclear weapons tests [Burbidge, 1957] [Wene, 1974] 
[Wene, 75] [Klapdor, 1981] [Meyer, 1989]. In the r-process, the large neutron fluxes, coupled with the availability of heavy target material, leads to multiple neutron capture events. This can produce neutron-rich nuclei all the way out to the neutron drip-line. These neutron-rich nuclei undergo beta decay which produces elements of higher atomic number. If this chain of beta decays were to continue, the very heavy actinides would be produced in relative amounts which are neither found in nature nor in nuclear weapons tests [Hoff, 1986] [Hoff, 1988]. It is believed that BDF depletes the beta decay chains. This reduces the production of higher atomic numbered elements [Burbidge, 1957] [Meyer, 1989]. The enhanced QECS associated with the decay of odd-proton, odd-neutron nuclei may reduce the relative amounts of the resulting even-proton, even-neutron daughters relative to odd-even or even-odd nuclei. However, reexamination of the available data by Hoff [Hoff, 1986] [Hoff, 1988] has shown that the predicted influence of BDF has been overestimated but is not negligible. The influence of delayed fission (DF) processes on the production and isotopic abundances of the actinide cosmochronometers can give important information applicable to determination of the age of the galaxy [Theilemann, 1983] [Yokoi, 1983] [Meyer, 1986] and its chemical evolution [Meyer, 1986] [Reeves, 1976].

DF also has the potential to greatly expand the number of nuclei whose fission properties can be measured; the nuclei in the actinide region have ground state fission halflives which are too long compared to their overall half-lives to allow detailed study. This is illustrated in Figure 2. The spontaneous fission half-lives in regions where ECDF is important are too long to make study practical. The delayed fission process makes possible the study of the fission properties of some of these ordinarily fission stable nuclides.

Of the most significance, however, is the possibility for detailed study of the highly deformed shape isomer in an out-of-beam environment. Excited states in the daughter are populated with the half-life of the EC parent. These half-lives can be of the order of minutes and thus allow sufficient time for transport of the nuclides out of the target system for subsequent collection and measurement. The ability to remove the reaction products from the irradiation area greatly reduces the experimental difficulties (background, etc.) and could make possible the study of the shape isomer (and the fission barrier) in unprecedented detail [Gregorich, 1991] (see Figure 3).

As early as 1972, Skobelev et al. [Skobelev, 1972] claimed that fissions observed in the light americium and neptunium region by Kuznetsov et al. [Kuznetsov, 1966] 
[Kuznetsov, 1967] were the result of ECDF. Fission decay in ${ }^{232}$ Am was confirmed by Habs et al. [Habs, 1978] in 1978. It was not until 1989, however, that Hall et al. [Hall, 1989B] provided direct evidence for ECDF in ${ }^{232} \mathrm{Am}$ by measuring the time correlation between the $\mathrm{EC} \mathrm{x}$-rays and the subsequent fission. In the measured coincidence spectra, other photopeaks appeared which may have resulted from transitions in the second potential well [Hall, 1992]. However, sufficient statistics were not available to confirm such a claim. The limited number of events did not eliminate the possibility that the unidentified photopeaks were random coincidences with prompt $\gamma$-rays from the fission fragments. All reports of ECDF are summarized in Table 1.

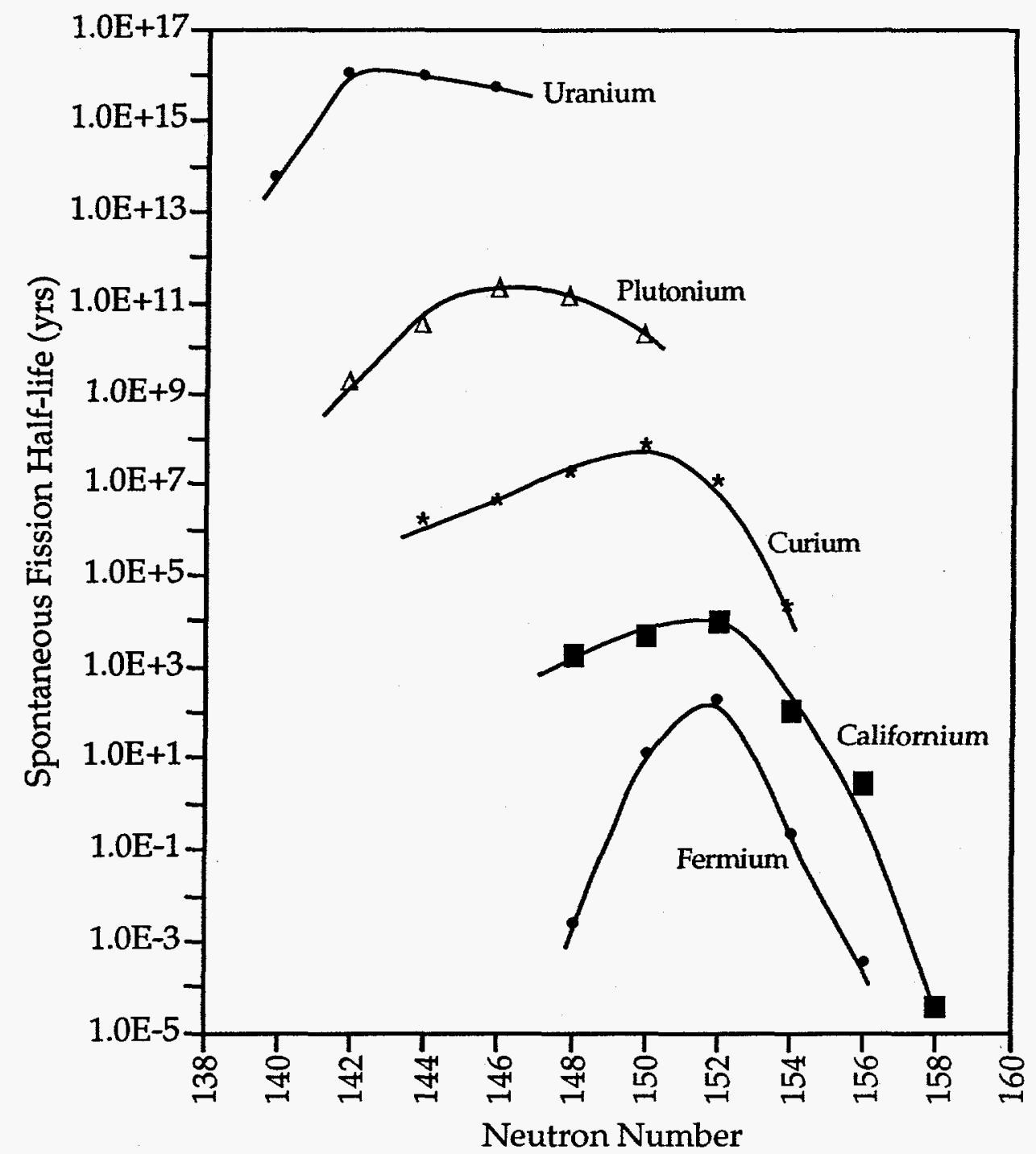

Plot of spontaneous fission half-lives for even-proton, even-neutron isotopes versus neutron number.

Figure 2. 
Delayed Fission Probability:

Experimentally, the probability of delayed fission, $\mathrm{PDF}_{\mathrm{DF}}$, is defined as the number of electron-capture decays which result in a fission, $\mathrm{N}_{\mathrm{ECDF}}$, divided by the total number of electron-capture decays, $N_{E C}$. This is illustrated by equation 1 . Typical values of $P_{D F}$ range from $10^{-2}$ to $10^{-8}$ (see Table 1). Any time the $\mathrm{P}_{\mathrm{DF}}$ approaches the height of the fission barrier, delayed fission becomes a possibility.

$$
P_{D F}=N_{E C D F} / N_{E C}
$$

Table 1. List of all reports of electron-capture delayed fission. aPDF is defined as the number of electron-capture decays which result in fission divided by the total number of electron-capture decays.

\begin{tabular}{|c|c|c|c|c|}
\hline Nuclide & Half-life & $\mathrm{PDF}^{\mathrm{a}}$ & $\begin{array}{l}\text { Production } \\
\text { Reaction }\end{array}$ & Reference \\
\hline $\mathrm{Md}-250$ & $52 \mathrm{sec}$ & $2 \times 10^{-4}$ & ${ }^{243} \mathrm{Am}\left({ }^{12} \mathrm{C}, 5 \mathrm{n}\right)$ & Gangrskii, 1980 \\
\hline Es-248 & $28 \mathrm{~min}$ & $3 \times 10^{-7}$ & ${ }^{238} \mathrm{U}\left({ }^{14} \mathrm{~N}, 4 \mathrm{n}\right)$ & Gangrskii, 1980 \\
\hline Es-246 & $8 \mathrm{~min}$ & $3 \times 10^{-5}$ & ${ }^{238} \mathrm{U}\left({ }^{14} \mathrm{~N}, 6 \mathrm{n}\right)$ & Gangrskii, 1980 \\
\hline Es-244 & $37 \mathrm{sec}$ & $\approx 10^{-4}$ & ${ }^{235} \mathrm{U}\left({ }^{14} \mathrm{~N}, 5 \mathrm{n}\right)$ & Gangrskii, 1980 \\
\hline Es-242? & $\approx 25 \mathrm{sec}$ & $1.4 \times 10^{-2}$ & ${ }^{205} \mathrm{Tl}\left({ }^{40} \mathrm{Ar}, 3 \mathrm{n}\right)$ & Hingmann, 1985 \\
\hline Bk-240 & $4 \mathrm{~min}$ & $\approx 10^{-5}$ & ${ }^{232} \operatorname{Th}\left({ }^{14} \mathrm{~N}, 6 \mathrm{n}\right)$ & Gangrskii, 1980 \\
\hline Am-236 & $3.7 \mathrm{~min}$ & $2.5 \times 10^{-8}$ & ${ }^{237} \mathrm{~Np}(\alpha, 5 n)$ & Hall, 1989D \\
\hline Am-234 & $\begin{array}{l}2.6 \mathrm{~min} \\
2.32 \mathrm{~min}\end{array}$ & $\begin{array}{l}\approx 10^{-5} \\
6.6 \times 10^{-5}\end{array}$ & $\begin{array}{l}{ }^{230} \mathrm{Th}\left({ }^{10} \mathrm{~B}, 6 \mathrm{n}\right) \\
{ }^{237} \mathrm{~Np}(\alpha, 7 \mathrm{n})\end{array}$ & $\begin{array}{l}\text { Skobelev, } 1972 \\
\text { Hall, 1990A }\end{array}$ \\
\hline Am-232 & $\begin{array}{l}1.4 \mathrm{~min} \\
0.92 \mathrm{~min} \\
1.31 \mathrm{~min}\end{array}$ & $\begin{array}{l}\approx 10^{-2} \\
1.3 \times 10^{-2} \\
6.9 \times 10^{-4}\end{array}$ & $\begin{array}{l}230_{\operatorname{Th}}\left({ }^{10} \mathrm{~B}, 8 \mathrm{n}\right) \\
237_{\mathrm{Np}}(\alpha, 9 \mathrm{n}) \\
{ }^{237} \mathrm{~Np}(\alpha, 9 \mathrm{n})\end{array}$ & $\begin{array}{l}\text { Skobelev, } 1972 \\
\text { Habs, } 1978 \\
\text { Hall, 1990B }\end{array}$ \\
\hline Np-228 & $60 \mathrm{sec}$ & $\approx 10^{-3}$ & ${ }^{209} \mathrm{Bi}\left({ }^{22} \mathrm{Ne}, 3 \mathrm{n}\right)$ & Skobelev, 1972 \\
\hline T1-180 & $0.7 \mathrm{sec}$ & $\approx 10^{-6}$ & ${ }^{144} \mathrm{Sm}\left({ }^{40} \mathrm{Ca}, \mathrm{p} 3 \mathrm{n}\right)$ & Lazarev, 1987 \\
\hline
\end{tabular}

Theoretically, $\mathrm{P}_{\mathrm{DF}}$, depends on several factors. These include the QEC; the excitation energy, E, of the levels populated in the daughter by the electron-capture; a 
transition probability function, $\mathrm{W}_{\mathrm{EC}}(\mathrm{E})$; and the ratio of the fission decay width, $\Gamma_{\mathrm{f}}(\mathrm{E})$, to the total decay width of the excited daughter, $\Gamma_{t}(E)$. The total decay width is the sum of decay widths for all possible decay modes. For our purposes, it is assumed that only gamma and fission decay modes compete, therefore $\Gamma_{\mathfrak{t}}(E)=\Gamma_{\gamma}(E)+\Gamma_{f}(E)$ where $\Gamma_{\gamma}(E)$ is the gamma decay width. This is not completely correct because other factors including neutron emission should be considered, if the decay energy is larger than the neutron binding energy. To calculate the $P_{D F}$, these factors must be integrated over the total energy available for the decay, QEC, and normalized to the total number of electron-capture decays. The $\mathrm{P}_{\mathrm{DF}}$ is given from theoretical considerations in functional form as equation 2.

$$
P_{D F}=\frac{\int_{0}^{Q_{E C} W_{E C}}(E) \frac{\Gamma_{f}}{\Gamma_{t}}(E) d E}{\int_{0}^{Q_{E C} W_{E C}}(E) d E}
$$

Approximations can be included in equation 2 for the transition probability function, and the decay widths in the following manner.

The gamma decay width term, $\Gamma_{\gamma}(\mathrm{E})$, can be approximated [Gangrskii, 1980] from the probability for gamma transitions, $\mathrm{P}_{\gamma}$, as equation 3 .

$$
\Gamma_{\gamma}(E)=\frac{P_{\gamma}}{2 \pi \rho(E)}=\frac{C_{\gamma} \Theta^{4} e^{(E / \Theta)}}{2 \pi \rho(E)}
$$

In equation $3, \rho(E)$ is the nuclear level density, $C_{\gamma}$ is a constant with the value $9.7 \times 10^{-7}$ $\mathrm{MeV}^{-4}$ and $\Theta$ is the nuclear temperature $(0.5-0.6 \mathrm{MeV})$. The nuclear temperature can be calculated from formulas such as those in [Swiatecki, 1983].

The fission width term, $\Gamma_{\mathrm{f}}(\mathrm{E})$, can be derived from the penetrability of the fission barrier. This yields equation 4 .

$$
\Gamma_{f}(E)=\frac{P_{f}}{2 \pi \rho(E)}
$$

In equation $4, \mathrm{P}_{\mathrm{f}}$ is the penetrability of the entire two humped fission barrier (see Figure 1). 
Calculation of the penetrability of the fission barrier in the actinides is difficult because the fission barriers are complex [Habs, 1978] [Gangrskii, 1980]. It is common to simplify the problem by assuming all nuclear motion in the second potential well is damped. This has the effect of allowing time for all states populated in the second potential well to gamma decay to the ground state in the second well before fission can occur. Calculating the penetrability of the outer barrier from this single level in the second well is reduced to a calculation involving a simple transmission coefficient. The penetrability of the inner barrier can be calculated using a parabolic barrier such as that used by Hill and Wheeler [Hill, 1953]. The penetrability of the fission barrier is then the product of the transmission coefficient from the lowest state in the second well through the outer barrier and the penetrability of the inner barrier. With inclusion of the Hill-Wheeler formalism for $\mathrm{P}_{\mathrm{f}}, \Gamma_{\mathrm{f}}(\mathrm{E})$ becomes:

$$
\Gamma_{\mathrm{f}}(\mathrm{E}) \approx \frac{\mathrm{R}_{\mathrm{B}}}{2 \pi \rho(\mathrm{E})}\left[1-\exp \left(\frac{2 \pi\left(\mathrm{B}_{\mathrm{f}}-\mathrm{E}\right)}{\hbar \omega_{\mathrm{f}}}\right)\right]^{-1}
$$

In equation $5, \mathrm{R}_{\mathrm{B}}$ is the transmission coefficient for decay from the lowest state in the second potential well through the outer fission barrier, $\mathrm{B}_{\mathrm{f}}$ is the inner barrier height, $\hbar \omega_{\mathrm{f}}$ is the energy associated with the curvature of the inner fission barrier, and $\mathrm{E}$ is the excitation energy of the state from which the decay proceeds. Because the lowest state in the second well can either fission or $\gamma$-decay back to the first well, the factor $R_{B}$ is really comprised of the ratio of transmission coefficients for the lowest level in the second well for tunneling through the outer barrier to that for $\gamma$-decay back to the first potential well.

The transition probability function in equation $2, \mathrm{~W}_{\mathrm{EC}}(\mathrm{E})$, consists of the product of the integrated Fermi function (dictates the rate of the $\mathrm{EC}$ transition) and a beta strength function, $\zeta_{\beta}$ (dictates the distribution of states available for the $\mathrm{EC}$ to populate). This product accounts for population of excited states in the daughter. The Fermi function for allowed electron-capture transitions can be approximated as $\left(\mathrm{QEC}_{\mathrm{EC}}-\mathrm{E}\right)^{2}$, where $\mathrm{E}$ represents the residual excitation energy in the daughter. The difference between $\mathrm{QEC}_{\mathrm{EC}}$ and $E$ is the transition energy of the electron-capture decay; The electron binding energy is ignored in this approximation. 
Use of the beta strength function, $\zeta_{\beta}$, to calculate the probability of population of states is justified because $\zeta_{\beta}$ is related to averaged properties of the nuclear decay which do not depend on the detailed structure of the levels. This treatment is only valid at relatively high excitation energies in the daughter, i.e. in the region where $\mathrm{P}_{\mathrm{DF}}$ becomes important. Klapdor et al. pointed out that low lying structure may affect $\zeta_{\beta}$ and thus the $P_{D F}$. This can be illustrated with the following example.

Example 1: If a nucleus has a large $\mathrm{QEC}_{\mathrm{EC}}$ relative to the height of the fission barrier in the daughter, the $\mathrm{P}_{\mathrm{DF}}$ would be expected to be large. However, if decay to the ground state of the daughter is superallowed (no net change in spin or parity), no high-energy states would be populated from which delayed fission could occur. The beta strength to high energy states would be zero. The PDF would be zero in this case.

The beta strength function has been approximated in several ways. It can be taken as proportional to the nuclear level density [Wene, 1974] [Shalev, 1977]; it can be generated from the gross theory of beta decay [Kodama, 1975]; or it can be taken as constant above a certain energy [Kratz, 1973] [Hornshøj, 1975]. Meyer et al. [Meyer, 1989] have presented a new method to calculate $\zeta_{\beta}$ which includes a specific treatment of nuclear deformation. This is important because the distribution of states is influenced significantly through mixing caused by deformation.

The model of Meyer et al. [Meyer, 1989] calculates beta strength functions by treating nuclear deformation explicitly using the random-phase approximation to mix deformed states; the Gamow-Teller (GT) interaction mixes states and significantly alters the beta-decay properties. The GT interaction provides more low energy states for the betadecay to populate and thus influences the $\mathrm{PDF}$.

To calculate effects such as that given in example 1 would require a knowledge of the low-lying structure of $\zeta_{\beta}$. Equation 2 is applicable for a qualitative understanding of the factors involved in determining the delayed fission probability. A more comprehensive approach would include factors to account for detailed structure of the fission barrier, 
nuclear deformation, and a better expression for $\zeta_{\beta}$ which more realistically models the true level scheme of the electron-capture daughter.

It may be necessary to include additional factors in equation 2 which account for fission from levels not directly populated by the electron-capture but by low energy gamma transitions from higher energy states. Because the fission width decreases very rapidly with decreasing excitation energy, it is likely that this factor is extremely small, at least for transitions in the first potential well. It becomes even less significant by realization that low energy gamma transitions to states where the fission width is still appreciable would be several orders of magnitude slower than high energy gamma transitions to states well inside the first potential well [Weisskopf, 1951].

With the use of the above approximations for $\mathrm{W}_{\mathrm{EC}}$ and $\Gamma_{t}$, equation 2 becomes equation 6 . This expression for the delayed fission probability includes the cut-off energy approximation, C, of Kratz et al. [Kratz, 1973] for the beta strength function below which the $\mathrm{PDF}_{\mathrm{DF}}$ is zero. Because the beta strength function above the cut-off energy is assumed to be constant and is present in both the numerator and denominator of equation 6 , it is simply divided out of the expression. The cut-off energy below which the beta strength function is zero is given by $\mathrm{C}=26 \mathrm{~A}^{-1 / 2} \mathrm{MeV}$ [Kratz, 1973], where ' $\mathrm{A}$ ' is the mass number of the fissioning species.

$$
P_{D F} \approx \frac{\int_{C}^{Q_{E C}}\left(Q_{E C}-E\right)^{2} \frac{\Gamma_{f}}{\Gamma_{f}+\Gamma_{\gamma}}(E) d E}{\int_{C}^{Q_{E C}}\left(Q_{E C}-E\right)^{2} d E}
$$

The denominator of equation 6 is easily evaluated yielding equation 7. This equation has an exponential dependence on the difference between the QEC and the fission barrier, illustrating the requirement that the QEC must be of the same order as the height of the fission barrier for delayed fission to become a significant mode of decay.

$$
P_{D F} \approx \frac{\int_{C}^{Q_{E C}}\left(Q_{E C}-E\right)^{2} \frac{\Gamma_{f}}{\Gamma_{f}+\Gamma_{\gamma}}(E) d E}{\frac{1}{3}\left(Q_{E C}-26 A^{-1 / 2}\right)^{3}}
$$


Equation 7 includes factors for the barrier height and curvature in the $\Gamma_{\mathrm{f}}(\mathrm{E})$ term given in equation 5 , but it is not possible to calculate either of these quantities explicitly without specific information about the shape isomer. As a general model, however, equation 7 is quite useful.

\section{Delayed Fission and the Shape Isomer:}

The recent observation of a time correlation between the electron-capture $\mathrm{x}$-rays and the subsequent fission [Hall, 1989B] has shed new light on the study of shape isomerism in the actinides. Because population of states in the fissioning species occurs with the halflife of the electron-capture parent, it is possible to separate the activity from the high background associated with typical in-beam studies. If excited states in the second well are populated and motion in the second well is damped, it should be possible to detect specific gamma transitions occurring inside the second well. With the availability of high-geometry $\gamma$-ray detector arrays, delayed fission may be used as a sensitive probe of the inner and outer fission barriers [Gregorich, 1991] (see Figure 3).

To accomplish a detailed analysis of the fission barrier the level scheme in the second potential well must be determined. This can be accomplished by measuring $\gamma$-rays in coincidence with delayed fissions. The large $\gamma$-ray multiplicity associated with the highly excited fission fragments will produce a background problem. However, with sufficient statistics, any transitions in the second well should be observable above this $\gamma$-ray continuum. The recently published results on the ECDF of ${ }^{232} \mathrm{Am}$ [Hall, 1990B] show this isotope as a promising candidate for such a study.

Information abọt the levels in the first potential well can be obtained through an $\mathrm{x}$ ray $-\gamma$-ray coincidence experiment. Data from a $\gamma$-ray- $\gamma$-ray coincidence experiment between a transition $\gamma$-ray in the second well and a $\gamma$-ray from the second to the first potential well will yield the probability for gamma decay back to the first well. It may also yield the excitation energy associated with the ground state in the second potential well. Studies by Bjørnholm et al. have shown that the excitation energy associated with the ground state of the second well is of order $3 \mathrm{MeV}$ [Bjørnholm, 1967] in the Pu region. 


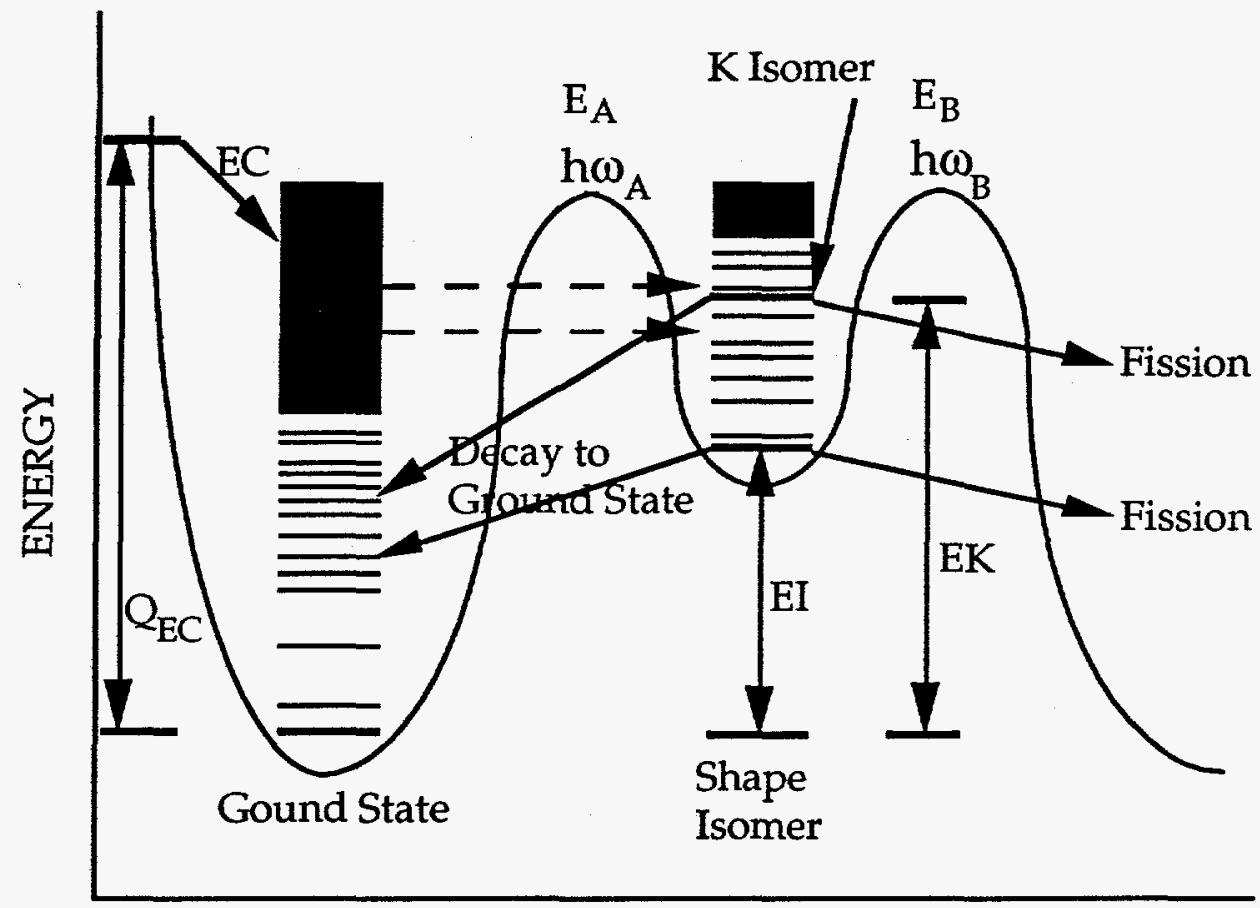

\section{DEFORMATION}

Figure 3. Schematic of how ECDF can be used to probe the fission barrier. $\mathrm{EI}$ is the energy of the lowest level in the shape isomer; EK is the energy of the $\mathrm{K}$-isomeric level in the second well; QEC is the electron capture Qvalue.

Other information about the second well, such as the fission probability from the ground state in the second well, may be obtainable through the x-ray-fission coincidence technique. With this information, it is possible to calculate the relative height and widths of both the inner and outer fission barriers from the ratio of the fission probability to the probability for $\gamma$-decay back to the first potential well.

If the $\mathrm{QEC}_{\mathrm{EC}}$ is sufficiently large, it may be possible to populate a $\mathrm{K}$-isomeric state in the second potential well (gamma transitions between different rotational bands are hindered. If the states are sufficiently hindered, a $\mathrm{K}$-isomer results). These levels are about 1-2 MeV above the lowest state and are typically several orders of magnitude longer lived than the timing resolution of a Ge $\gamma$-ray detector, which is about 10-20 nsec. Determination of the $\mathrm{K}$-isomeric lifetime and the probability for fission from this level will 
yield the penetrability of the outer barrier at the $\mathrm{K}$ isomer energy. The lifetime of the ground state in the shape isomer may be obtained with techniques such as decay in flight. Determination of the gamma transition probability from the $\mathrm{K}$ isomer back to the first potential well and the $\mathrm{K}$-isomeric lifetime will permit calculation of the penetrability of the inner barrier at the $\mathrm{K}$ isomer energy. These studies can be accomplished with $\gamma$-ray-fission and $\gamma$-ray- $\gamma$-ray coincidence techniques. The energy of the $\mathrm{K}$-isomeric state can be determined by measuring its decay to either the lowest level in the shape isomer or to the ground state in the first potential well.

The absolute heights and widths (curvature) of the inner and outer barriers can be determined from the ratios of the penetrabilities of the inner and outer barriers from the lowest level in the shape isomer and the $\mathrm{K}$-isomeric level. This is critical to the refinement of the existing nuclear models because absolute measurements of the fission barrier have never been achieved.

The americium region was chosen for several reasons. First, ECDF branches have been reported in the americium [Habs, 1978] [Hall, 1990A, B] [Liu, 1983] region, previously. Second, as stated above, the $\mathrm{Q}_{\mathrm{EC}}$ must be of the same order as the height of the fission barrier for ECDF to become an important decay mode. Fission barriers in the actinide region have been shown to be of the order of $5 \mathrm{MeV}$ [Habs, 1978]. Therefore, isotopes with a $\mathrm{Q}_{\mathrm{EC}}$ value larger than about $4 \mathrm{MeV}$ should begin to have measurable $\mathrm{ECDF}$ branches. Nuclides with sufficient $\mathrm{Q}_{\mathrm{EC}} \mathrm{S}$ are found in the very neutron-deficient oddproton, odd-neutron nuclei. Odd-odd nuclei are preferable because of the enhanced electron-capture $Q$-values associated with decay to their more stable even-even daughters. Very light even mass number americium isotopes which are odd-odd fit these criteria. Some isotopes for which delayed fission probabilities have been reported as well as other potential candidates are shown in Figure 4. The Light Ion Multiple Target system (LIM) [Hall, 1989A] makes it possible to use many targets, thereby increasing the production rates sufficiently to make study of isotopes in this region (which have relatively small production cross-sections) practical using reactions of light ions with actinide targets. Light ion bombardments are preferable over heavy ions because of the generally larger reaction cross sections, and higher available beam intensities. 


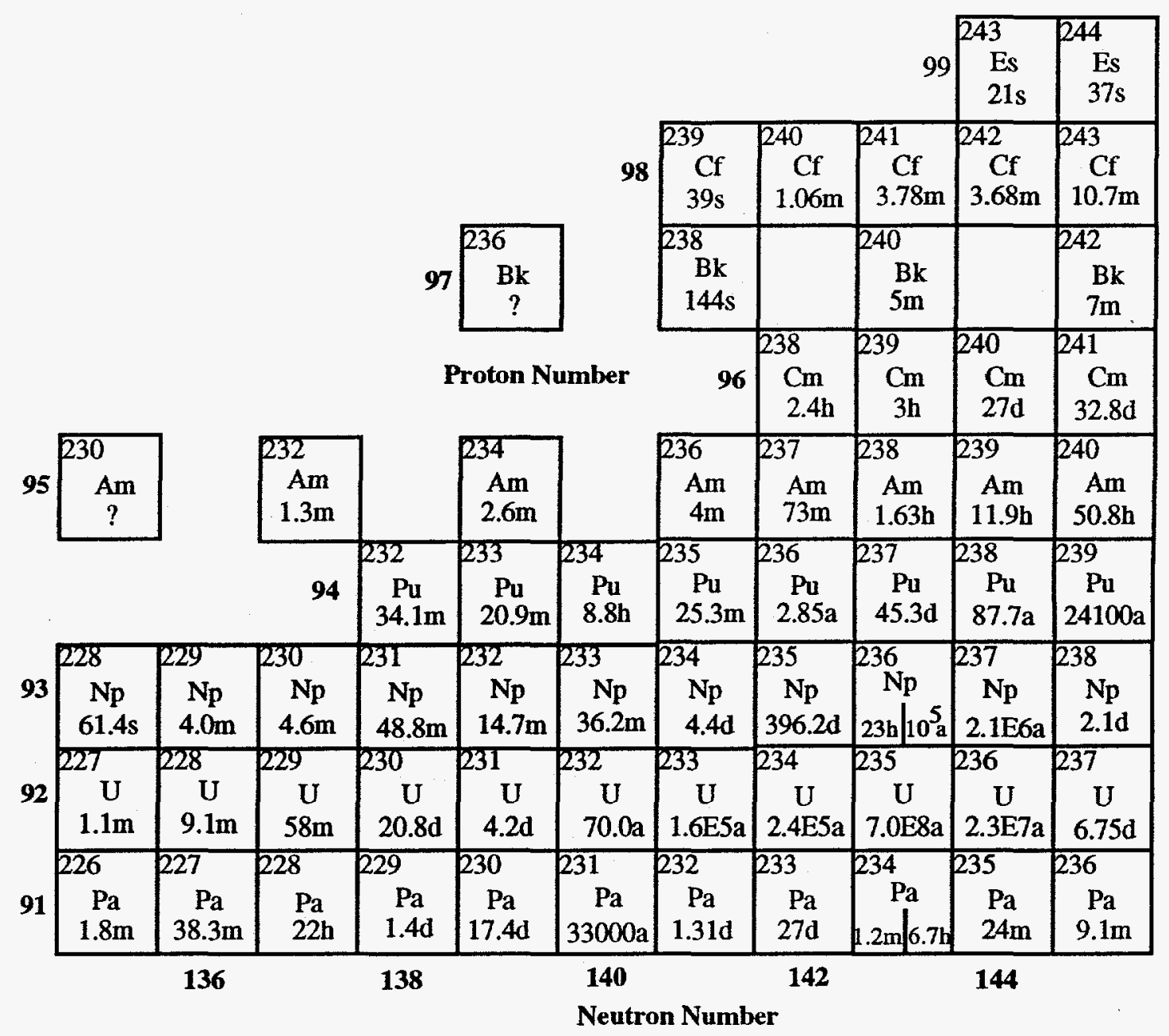

Figure 4. Actinide region where isotopes may have significant delayed fission probabilities. Odd proton-odd neutron nuclei have larger $\mathrm{Q}_{\mathrm{EC}} \mathrm{s}$ and therefore should have larger PDF branches than even-odd or odd-even nuclei.

The Automated X Ray-Fission Coincidence Apparatus:

An advanced radiation measurement system is required to study the ECDF process in sufficient detail to extract fission barrier information. We have designed and constructed an $\mathrm{x}$-ray-fission coincidence apparatus for use with the Gammasphere high efficiency gamma-ray detector array at Lawrence Berkeley National Laboratory (LBNL). The x-rayfission coincidence apparatus detection station consists (see Figure 5) of two surface barrier detectors (for detection of fission fragments) and two high-purity Ge (HPGe) planar x-ray detectors (for measurement of $\mathrm{x}$-rays and low-energy gamma rays). The detection station is placed inside Gammasphere at the 88-Inch Cyclotron at LBNL and used in conjunction 
with Gammasphere to measure the $\mathrm{x}$-rays and gamma-rays resulting from the ECDF process. Nuclides of interest are produced in the high-flux irradiation area at the LBNL 88Inch Cyclotron and transported to the detector station via salt $(\mathrm{KCl})$ aerosols carried in helium gas. We have designed (and are currently upgrading) an automated sample collection and handling system to collect the $\mathrm{KCl}$ aerosols (and produced radionuclides) on thin polypropylene foils (to minimize fission fragment energy degradation) and position the samples inside the $\mathrm{x}$-ray-fission coincidence apparatus for measurement.

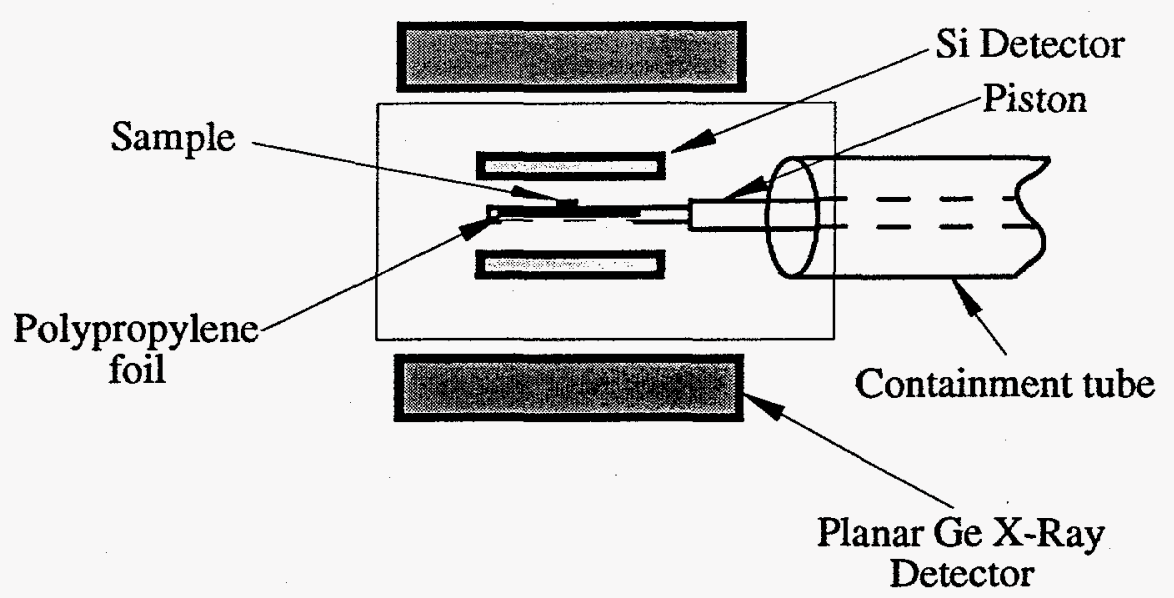

Figure 5. X Ray-Fission coincidence detector station detector schematic (not to scale).

A major portion of the design work involved development of a sample handling mechanism to first collect and then position the radioactive $\mathrm{KCl}$ aerosols in Gammasphere without risk of contamination of the Gammasphere detector array. Engineering difficulties included development of $x$-ray detectors capable of extending the extreme length required to reach the center of the Gammasphere array, and development of a positioning system sufficiently fast as to be able to get samples in and out of Gammasphere in real-time yet gentle enough so as to not disturb the extremely thin polypropylene foil and risk contaminating the detector array.

The sample changing assembly consists of a vacuum pump connected to the $\mathrm{KCl}$ aerosol collection assembly, a supply stack of polypropylene foils $\left(\approx 40 \mu \mathrm{g} / \mathrm{cm}^{2}\right)$, an empty stack for the used sample foils, and a worm driven piston that positions the samples inside and removes the measured samples from the $\mathrm{x}$-ray-fission coincidence detector station. 
The collection system is controlled by a Mycom 210, 2 axis controller connected to a PC. The worm drive moves the piston (and polypropylene foil with the nuclides of interest) into Gammasphere through a plastic tube to the $x$-ray-fission coincidence station. The surface barrier detectors ( $400 \mathrm{~mm}^{2}$ area, transmission mounted, $500 \mu \mathrm{m}$ depletion depth) of the $\mathrm{x}$ ray-fission coincidence detector station and sample are contained in a thin mylar and aluminum containment vessel and the HPGe x-ray detectors ( $36 \mathrm{~mm}$ diameter, $13 \mathrm{~mm}$ thick, Be window) are positioned outside the containment vessel. Helium gas is passed through the containment vessel to minimize fission fragment energy degradation. The surface barrier detectors are approximately $0.5 \mathrm{~cm}$ from the sample, with the bottom detector somewhat farther because of the thickness of the sample support piston and the fact that the $\mathrm{KCl}$ deposit is on the top surface of the polypropylene (see Figure 5).

Development of the $\mathrm{x}$-ray detectors required significant engineering on the part of EG\&G Instruments to minimize microphonic noise that could result from mechanical vibration of the extremely long cryostat/coldfinger; The cryostat/coldfinger extends 30 inches from the outside of the Gammasphere array to the center which is nearly two times longer than previously developed by EG\&G for planar detectors. Even with the extreme length, these detectors have better than $\approx 550 \mathrm{eV}$ resolution at $122 \mathrm{keV}$. Figure 6 is a schematic of the $\mathrm{x}$-ray detectors. Figure 7 is a schematic of the entire $\mathrm{x}$-ray-fission coincidence apparatus detection station and sample changing assembly.

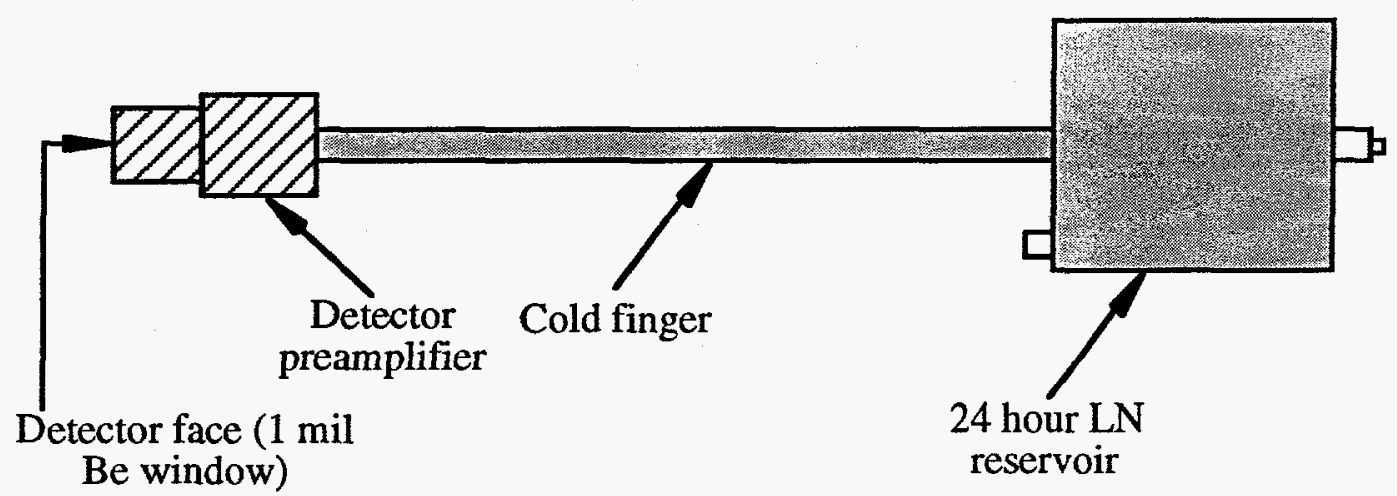

Figure 6. X Ray detector with 30-inch cold-finger (approximately to scale). The $\mathrm{x}$-ray detectors are mounted on the structural cage of Gammasphere and reach in through the BGO shielding to the center of Gammasphere, approximately 30 inches. 


\section{Current Status:}

LBNL is currently upgrading the worm drive to a pulley system. This is because the worm drive, while very reliable and nearly impossible to jam, is too slow (approximately 60 seconds to transfer the collected $\mathrm{KCl}$ aerosols to the piston and move the sample into position). The aerosol transport time from the high-flux bombardment area to the collection site is sufficiently large (nearly $100 \mathrm{~m}$ ) that a significant decay time results. The time required to get the sample in the detector system must be minimized to make detection of the 1-2 minute half-life nuclides practical. The worm drive arrangement is too slow to permit effective measurement of short-lived nuclides. This necessitated the upgrade. The expected positioning time of the new pulley system is of the order of 15 seconds and is a significant improvement over the worm driven system.

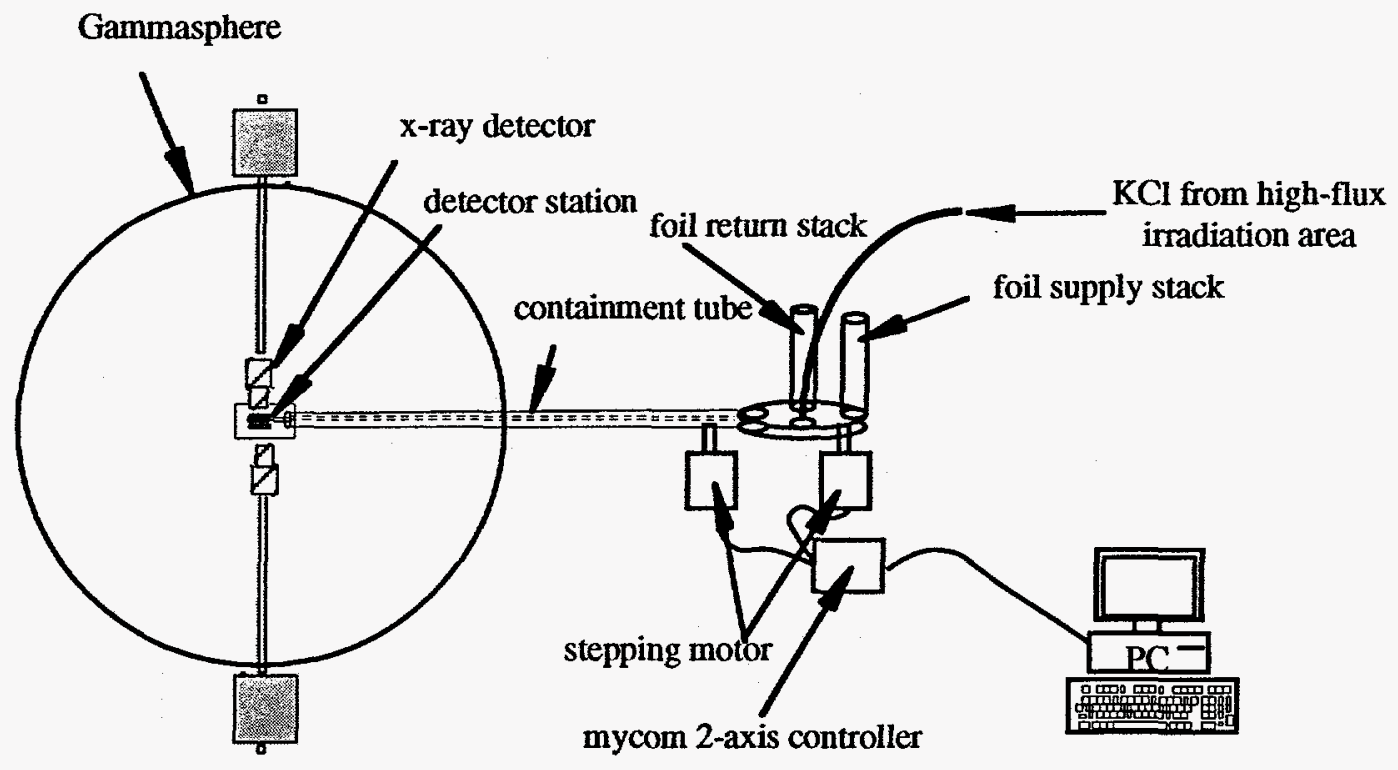

Figure 7. Schematic of the complete $x$-ray-fission coincidence apparatus with the worm driven sample changing system. This system is being modified to replace the worm drive system with a pulley system to decrease the sample handling time prior to placement in the detector assembly.

A series of collaborative experiment between LLNL, LBNL, and LANL utilizing various components of the $\mathbf{x}$-ray-fission coincidence apparatus to measure $\mathbf{x}$-rays and gamma-rays in the decay of a stationary ${ }^{252} \mathrm{Cf}$ source were performed to test various components of the $\mathrm{x}$-ray-fission coincidence apparatus. The test experiments have been 
completed and the data is currently being analyzed by LBNL. The components tested performed better than expected (e.g., the $\mathrm{x}$-ray detectors performed better than expected with no evidence of microphonic noise that would reduce the photon energy resolution).

LBNL expects to begin experiments to measure the ECDF decay of ${ }^{232} \mathrm{Am}$ are expected to begin later this year

The ability to position $\mathrm{x}$-ray detectors inside of Gammasphere near the sample has proven to be a significant resource to scientists studying ECDF and other nuclear decay properties with the Gammasphere array. Completion of this automated changer and detection system represents a major achievement in expanding the capabilities of Gammasphere for high-flux irradiations; Gammasphere cannot accommodate high-flux inbeam studies due to significant damage to the detector array from the neutron flux generated in such irradiations. The ability to position low-energy photon detectors ( $\mathrm{x}$-ray detectors) inside the Gammasphere array has also greatly expanded the capabilities of Gammasphere because the array is not capable of detecting low energy gamma-rays or $\mathrm{x}$-rays (less than about $80 \mathrm{keV}$ in energy) because of attenuation of these photons in the air between the center of the array and the detectors and the presence of a "thick" detector window and BGO shield. Since gamma-ray transitions between the near-ground rotational states are in the $20-100 \mathrm{keV}$ energy range, the ability to use low-energy photon detectors in conjunction with Gammasphere has proven very useful.

Publications and Presentations:

S. A. Kreek, "Delayed Fission and Accelerated Analyses," Nuclear Chemistry Division, Lawrence Livermore National Laboratory, Livermore, CA, (1994).

S. A. Kreek, H. L. Hall, K. E. Gregorich, R. A. Henderson, J. D. Leyba, K. R. Czerwinski, B. Kadkhodayan, M. P. Neu, C. D. Kacher, T. M. Hamilton, M. R. Lane, E. R. Sylwester, A. Türler, D. M. Lee, M. J. Nurmia, and D. C. Hoffman, "ElectronCapture Delayed Fission Properties of 238Bk," Physical Review C 46(4), 1859 (1994).

S. A. Kreek, H. L. Hall, K. E. Gregorich, R. A. Henderson, J. D. Leyba, K. R. Czerwinski, B. Kadkhodayan, M. P. Neu, C. D. Kacher, T. M. Hamilton, M. R. Lane, E. R. Sylwester, A. Türler, D. M. Lee, M. J. Nurmia, and D. C. Hoffman, "ElectronCapture Delayed Fission Properties of 228Np," Physical Review C 50(5), 2288 (1994). References:

[Hall, 1990A] H.L. Hall et al., Phys. Rev C 41, 618 (1990).

[Burbidge, 1957] E. M. Burbidge, G. R. Burbidge, W. A. Fowler and F. Hoyle, Rev. Mod. Phys. 29, 547 (1957).

[Wene, 1974] C.-O. Wene and S.A.E. Johansson, Phys. Scripta 10A, 156 (1974).

[Wene, 1975] C.-O. Wene, Astron. \& Astrophis. 44, 233 (1975). 
[Klapdor, 1981] H.V. Klapdor et al., Z. Physik A 299, 213 (1981).

[Meyer, 1989] B.S. Meyer et al., Phys. Rev. C 39, 1876 (1989).

[Hoff, 1986] R.W. Hoff, Weak and Electromagnetic Interactions in Nuclei, (H.V. Klapdor, ed., Springer-Verlag Publishers, Heidelburg, 1986) p.207.

[Hoff, 1988] R.W. Hoff, Inst. Phys. Conf. Ser. No. 88; J. Phys. G: Nucl. Phys. 14(Suppl.): S343-56 (1988).

[Theilemann, 1983] F.-K. Theilemann, J. Metzinger and H.V. Klapdor, Astron, Astrophys. 123, 162(1983).

[Yokoi, 1983] K. Yokoi, K. Takahashi and M. Arnould, Astron. Astrophys. $117,65(1983)$.

[Meyer, 1986] B.S. Meyer and D.N. Schramm, Astrophys J. 311, 406(1986).

[Reeves, 1976] H. Reeves and O. Johns, Astrophys. J., 206, 958(1976).

[Gregorich, 1991] K.E. Gregorich, private communication, 1991.

[Skobelev, 1972] N.K. Skobelev, Yad Fiz. 15, 444 (1972) [Sov. J. Nucl. Phys. 15, 249 (1972)].

[Kuznetsov, 1966] V.I. Kuznetsov, N.K. Skobelev and G.N. Flerov, Yad Fiz. 4, 279 (1966) [Sov. J. Nucl. Phys. 4, 202 (1967)].

[Kuznetsov, 1967] V.I. Kuznetsov, N.K. Skobelev and G.N. Flerov, Yad Fiz. 5, 271 (1967) [Sov. J. Nucl. Phys. 5, 191 (1967)].

[Habs, 1978] D. Habs, H. Klewe-Nebenius, V. Metag, B. Neumann and H. J. Specht, Z. Physik A 285, 53 (1978).

[Hall, 1989B] H.L. Hall et al., Phys. Rev. Let. 63, 2548 (1989).

[Hall, 1992] H.L. Hall and D.C. Hoffman, Ann. Rev. Nucl. and Particle Sci., 42, 147-175 (1992).

[Gangrskii, 1980] Yu. P. Gangrskii, M. B. Miller, L. V. Mikhailov and I. F. Kharisov, Sov. J. Nucl. Phys. 31, 162 (1980).

[Swiatecki, 1983] W.J. Swiatecki, Aust. J. Phys. 36, 641-648, 1983.

[Hill, 1953] D.L Hill and J.A. Wheeler, Phys. Rev. 89, 1102, (1953).

[Wene, 1974] C.-O. Wene and S.A.E. Johansson, Phys. Scripta 10A, 156 (1974).

[Shalev, 1977] S. Shalev and G. Rudstam, Nucl. Phys. A275, 76 (1977).

[Kodama, 1975] T. Kodama and K. Takahashi, Nucl. Phys. A239, 489 (1975).

[Kratz,1973] K.L. Kratz and G. Hermann, Z. Physik 263, 435 (1973).

[Hornshøj, 1975] P. Hornshøj et al., Nucl. Phys A239, 15 (1975).

[Weisskopf, 1951] V.F. Weisskopf, Phys. Rev, 83,1073(1951).

[Hall, 1990B] H.L. Hall et al., Phys. Rev. C 42, 1480 (1990).

[Bjørnholm, 1967] S. Bjørnholm, J. Borggreen, L. Westgaard and V.A. Karnaukhov, Nucl. Phys. A 95, 513 (1967).

[Liu, 1983] Yuan-Fang Liu, Cheng Luo, K. J. Moody, D. Lee, G. T. Seaborg, H. R. Von Gunten, J. Rad. Chem. 76(1), 119-124 (1983).

[Hall, 1989A] H.L. Hall et al., Nucl. Instr. Meth. A 276, 649 (1989). 